\title{
Nickel-catalyzed reductive alkylation of halogenated pyridines with secondary alkyl bromides
}

\author{
Huiyan Liu ${ }^{\text {a }}$, Zhuye Liang ${ }^{\text {b }}$, Qun Qian*a, Kunhua Lin*a \\ a Department of Chemistry, Shanghai University, 99 Shang-Da Road, Shanghai 200444, China \\ ${ }^{\mathrm{b}}$ College of Chemistry and Molecular Engineering, Zhengzhou University, 100 Science Road, Zhengzhou \\ 450001, China
}

\section{Table of Contents}

I. Experimental Section ................................S1-S2

II. Spectral Data for New Compounds.......................S2-S7

III. ${ }^{1} \mathrm{H}$ NMR and ${ }^{13} \mathrm{C}$ NMR spetra......................... S8-S20 


\section{$\underline{\text { I. Experimental Section }}$}

General Methods: All reactions were carried out under an atmosphere of nitrogen unless otherwise indicated. All NMR spectra were recorded on Bruker Avance $500 \mathrm{MHz}$ spectrometer at STP unless otherwise indicated. ${ }^{1} \mathrm{H}$ NMR and ${ }^{13} \mathrm{C}$ NMR chemical shifts are reported in $\delta$ units, parts per million (ppm) relative to the chemical shift of residual solvent. Reference peaks for chloroform in ${ }^{1} \mathrm{H}$ NMR and ${ }^{13} \mathrm{C}$ NMR spectra were set at $7.26 \mathrm{ppm}$ and 77.0 ppm, respectively. High-resolution mass spectra (HRMS) were obtained using a Agilent 7890-5975. Melting point was recorded on a micro melting point apparatus (X-4, YUHUA Co., Ltd, Gongyi, China). Column chromate -graphy was performed using silica gel 300-400 mesh (purchased from Qingdao-Haiyang Co. China) as the solid support.

Chemicals: The following anhydrous solvents were purchased from Acros: THF (99.5\%, stabilized), DMF (N,N- dimethylforma -mide, 99.8\%), DMA (N,N-dimethylacetamide, $99.5 \%$, extra pure). $\mathrm{NiBr}_{2}$ (Alfa Aesar), $\mathrm{Ni}(\mathrm{COD})_{2}$ (Strem). Zinc powder (Aladdin) was activated before use. Unless otherwise noted, all other reagents and starting materials were purchased from commercial sources and used without further purification. 4-bromo-1 -tosylpiperidine and 2-bromo-2,3-dihydro-1H-indene were synthesized according to the literature procedures.

General Procedure for alkylation of halogenated pyridines with 
secondary alkyl bromides: To a flame-dried Schlenk tube equipped with a magnetic stir bar was loaded alkyl bromide (0.15 mmol, $100 \mathrm{~mol} \%$, solid), halogenated pyridine (0.30 mmol, $300 \mathrm{~mol} \%$, solid), ligand (0.015 mmol, 10 mol\%), zinc powder (0.45 mmol, $300 \mathrm{~mol} \%)$. The tube was capped with a rubber septum and moved to a dry glove box, at which $\mathrm{NiBr}_{2}(0.015 \mathrm{mmol}$, $10 \mathrm{~mol} \%), \mathrm{MgCl}_{2}(0.15 \mathrm{mmol}, 100 \mathrm{~mol} \%)$ and $\mathrm{Bu}_{4} \mathrm{NBr}(0.075 \mathrm{mmol}, 50$ mol\%) were added. Then the tube was moved out of the glove box. Alkyl bromide (0.15 mmol, $100 \mathrm{~mol} \%$, liquid), halogenated pyridine $(0.30 \mathrm{mmol}$, $300 \mathrm{~mol} \%$, liquid), pyridine (0.15 mmol, $100 \mathrm{~mol} \%)$ and DMA(1.0 mL) were added via syringe. After the reaction mixture was allowed to stir overnight under $\mathrm{N}_{2}$ atmosphere at $30^{\circ} \mathrm{C}$, it was directly loaded onto a silica column without work-up. The residue in the reaction vessel was rinsed with small amount of DCM or eluent. Flash column chromatography provided the product as solid or oil.

\section{II .Spectral Data of New Compounds}

5-methyl-2-(1-tosylpiperidin-4-yl)pyridine (Table 2, entry 3), a white power solid. mp $184-186{ }^{\circ} \mathrm{C} .{ }^{1} \mathrm{H}$ NMR $\left(500 \mathrm{MHz}, \mathrm{CDCl}_{3}\right): \delta 8.32(\mathrm{~m}, 1 \mathrm{H})$, $7.66(\mathrm{~d}, J=8.3 \mathrm{~Hz}, 2 \mathrm{H}), 7.41(\mathrm{dd}, J=8.0 \mathrm{~Hz}, 2.0 \mathrm{~Hz}, 1 \mathrm{H}), 7.33(\mathrm{~d}, J=8.0$ $\mathrm{Hz}, 2 \mathrm{H}), 6.99(\mathrm{~d}, J=8.0 \mathrm{~Hz}, 1 \mathrm{H}), 3.91(\mathrm{~d}, J=11.6 \mathrm{~Hz}, 2 \mathrm{H}), 2.58(\mathrm{tt}, J=$ $12.1 \mathrm{~Hz}, 3.6 \mathrm{~Hz}, 1 \mathrm{H}), 2.44(\mathrm{~s}, 3 \mathrm{H}), 2.37(\mathrm{td}, J=12.0 \mathrm{~Hz}, 2.5 \mathrm{~Hz}, 2 \mathrm{H}), 2.28(\mathrm{~s}$, 
$3 \mathrm{H}), 2.02-1.93(\mathrm{~m}, 2 \mathrm{H}), 1.92-1.81(\mathrm{~m}, 2 \mathrm{H}) .{ }^{13} \mathrm{C} \mathrm{NMR}\left(125 \mathrm{MHz}, \mathrm{CDCl}_{3}\right): \delta$ $160.6,149.6,143.4,137.1,133.3,130.9,129.6,127.7,120.1,46.5,43.1$, 31.2, 21.5, 18.0. IR $\left(\mathrm{KBr}, \mathrm{cm}^{-1}\right) \vee 2993,2920,2838,1596,1459,1331,1243$, 1161,723 .

HRMS (ESI): $\mathrm{m} / \mathrm{z}\left(\mathrm{M}^{+}\right)$calcd for $\mathrm{C}_{18} \mathrm{H}_{22} \mathrm{~N}_{2} \mathrm{O}_{2} \mathrm{~S}: 330.1402$, found 330.1406.

4-methyl-2-(1-tosylpiperidin-4-yl)pyridine (Table 2, entry 4), a white power solid. mp 142-144 ${ }^{\circ} \mathrm{C} .{ }^{1} \mathrm{H}$ NMR $\left(500 \mathrm{MHz}, \mathrm{CDCl}_{3}\right): \delta 8.34(\mathrm{~d}, J=5.0$ $\mathrm{Hz}, 1 \mathrm{H}), 7.66(\mathrm{~d}, J=8.3 \mathrm{~Hz}, 2 \mathrm{H}), 7.33(\mathrm{~d}, J=8.0 \mathrm{~Hz}, 2 \mathrm{H}), 6.96-6.89(\mathrm{~m}$, 2H), $3.91(\mathrm{~d}, J=5.0 \mathrm{~Hz}, 2 \mathrm{H}), 2.57(\mathrm{tt}, J=12.0 \mathrm{~Hz}, 3.7 \mathrm{~Hz}, 1 \mathrm{H}), 2.44(\mathrm{~s}, 3 \mathrm{H})$, $2.36(\mathrm{td}, J=12.0 \mathrm{~Hz}, 2.5 \mathrm{~Hz}, 2 \mathrm{H}), 2.31(\mathrm{~s}, 3 \mathrm{H}), 2.01-1.95(\mathrm{~m}, 2 \mathrm{H}), 1.93$ $-1.82(\mathrm{~m}, 2 \mathrm{H}) .{ }^{13} \mathrm{C} \mathrm{NMR}\left(125 \mathrm{MHz}, \mathrm{CDCl}_{3}\right): \delta 163.2,148.8,147.5,143.3$, $133.1,129.4,127.5,122.5,121.4,46.3,43.2,31.0,21.4,20.9 . \mathrm{IR}\left(\mathrm{KBr}, \mathrm{cm}^{-1}\right)$ $v$ 2981, 2919, 2840, 1603, 1444, 1330, 1247, 1164, 726. HRMS (ESI): m/z $\left(\mathrm{M}^{+}\right)$calcd for $\mathrm{C}_{18} \mathrm{H}_{22} \mathrm{~N}_{2} \mathrm{O}_{2} \mathrm{~S}: 330.1405$. Found 330.1402.

2-(2,3-dihydro-1H-inden-2-yl)pyridine (Table 2, entry 5 and entry 6 ), a colorless oil. ${ }^{1} \mathrm{H}$ NMR (500 MHz, $\left.\mathrm{CDCl}_{3}\right): \delta 8.62-8.54(\mathrm{~m}, 1 \mathrm{H}), 7.61$ (td, $J=7.7 \mathrm{~Hz}, 1.9 \mathrm{~Hz}, 1 \mathrm{H}), 7.28-7.21(\mathrm{~m}, 3 \mathrm{H}), 7.20-7.16(\mathrm{~m}, 2 \mathrm{H}), 7.15-7.11(\mathrm{~m}$, 1H), 3.93-3.84 (m, 1H), $3.39(\mathrm{dd}, J=15.4 \mathrm{~Hz}, 8.4 \mathrm{~Hz}, 2 \mathrm{H}), 3.28(\mathrm{dd}, J=$ $15.5 \mathrm{~Hz}, 8.8 \mathrm{~Hz}, 2 \mathrm{H}) .{ }^{13} \mathrm{C} \mathrm{NMR}\left(125 \mathrm{MHz}, \mathrm{CDCl}_{3}\right): \delta 164.4,149.2,142.8$, $136.4,126.5,124.4,121.5,121.4,47.4,39.7 . \mathrm{IR}\left(\mathrm{KBr}, \mathrm{cm}^{-1}\right)$ v 3019,2934 2844, 1590, 1472, 1432, 743. HRMS (ESI): $\mathrm{m} / \mathrm{z}\left(\mathrm{M}^{+}\right)$calcd for $\mathrm{C}_{14} \mathrm{H}_{13} \mathrm{~N}$ : 
195.1048. Found 195.1045.

3-(2,3-dihydro-1H-inden-2-yl)pyridine(Table 2, entry 7), a yellow oil. ${ }^{1} \mathrm{H}$ NMR $\left(500 \mathrm{MHz}, \mathrm{CDCl}_{3}\right): \delta 8.63(\mathrm{~s}, 1 \mathrm{H}), 8.52(\mathrm{~d}, J=3.0 \mathrm{~Hz}, 1 \mathrm{H}), 7.63(\mathrm{~d}$, $J=8.0 \mathrm{~Hz}, 1 \mathrm{H}), 7.34-7.22(\mathrm{~m}, 5 \mathrm{H}), 3.82-3.70(\mathrm{~m}, 1 \mathrm{H}), 3.44(\mathrm{dd}, J=15.4 \mathrm{~Hz}$, $8.2 \mathrm{~Hz}, 2 \mathrm{H}), 3.12(\mathrm{dd}, J=15.5 \mathrm{~Hz}, 8.8 \mathrm{~Hz}, 2 \mathrm{H}) .{ }^{13} \mathrm{C} \mathrm{NMR}(125 \mathrm{MHz}$, $\left.\mathrm{CDCl}_{3}\right): \delta 149.0,147.7,142.3,140.7,134.2,126.7,124.4,123.4,42.7,40.6$. IR $\left(\mathrm{KBr}, \mathrm{cm}^{-1}\right) \vee 3022,2932,2845,1574,1479,1423,744$. HRMS (ESI): $\mathrm{m} / \mathrm{z}\left(\mathrm{M}^{+}\right)$calcd for $\mathrm{C}_{14} \mathrm{H}_{13} \mathrm{~N}: 195.1048$. Found 195.1045 .

4-(2,3-dihydro-1H-inden-2-yl)-2-methylpyridine (Table 2 , entry 8), a colorless oil. ${ }^{1} \mathrm{H}$ NMR $\left(500 \mathrm{MHz}, \mathrm{CDCl}_{3}\right): \delta 8.39(\mathrm{~d}, J=5.0 \mathrm{~Hz}, 1 \mathrm{H}), 7.28$ $-7.23(\mathrm{~m}, 2 \mathrm{H}), 7.22-7.17(\mathrm{~m}, 2 \mathrm{H}), 7.07(\mathrm{~s}, 1 \mathrm{H}), 7.00(\mathrm{~d}, J=5.0 \mathrm{~Hz}, 1 \mathrm{H})$, 3.70-3.55 (m, 1H), $3.36(\mathrm{dd}, J=15.4 \mathrm{~Hz}, 8.3 \mathrm{~Hz}, 2 \mathrm{H}), 3.06(\mathrm{dd}, J=15.5 \mathrm{~Hz}$, $8.5 \mathrm{~Hz}, 2 \mathrm{H}), 2.53(\mathrm{~s}, 3 \mathrm{H}) .{ }^{13} \mathrm{C} \mathrm{NMR}\left(125 \mathrm{MHz}, \mathrm{CDCl}_{3}\right): \delta 158.5,154.7$, $149.2,142.3,126.7,124.4,122.0,119.5,44.5,40.2,24.5 . \mathrm{IR}\left(\mathrm{KBr}, \mathrm{cm}^{-1}\right) v$ 3019, 2934, 2845, 1603, 1482, 1446, 746. HRMS (ESI): m/z $\left(\mathrm{M}^{+}\right)$calcd for $\mathrm{C}_{15} \mathrm{H}_{15} \mathrm{~N}: 209.1204$. Found 209.1206.

2-(2,3-dihydro-1H-inden-2-yl)-4-methylpyridine (Table 2, entry 9), a colorless oil. ${ }^{1} \mathrm{H}$ NMR $\left(500 \mathrm{MHz}, \mathrm{CDCl}_{3}\right): \delta 8.42(\mathrm{~d}, J=5.0 \mathrm{~Hz}, 1 \mathrm{H})$, 7.28-7.22 (m, 2H), 7.20-7.14 (m, 2H), $7.06(\mathrm{~s}, 1 \mathrm{H}), 6.96(\mathrm{~d}, J=5.0 \mathrm{~Hz}, 1 \mathrm{H})$, 3.92-3.77 (m, 1H), $3.76(\mathrm{dd}, J=15.4 \mathrm{~Hz}, 8.4 \mathrm{~Hz}, 2 \mathrm{H}), 3.27(\mathrm{dd}, J=15.6 \mathrm{~Hz}$, $9.0 \mathrm{~Hz}, 2 \mathrm{H}), 2.33(\mathrm{~s}, 3 \mathrm{H}) .{ }^{13} \mathrm{C} \mathrm{NMR}\left(125 \mathrm{MHz}, \mathrm{CDCl}_{3}\right): \delta 164.0,148.8$, 
$147.3,142.6,126.2,124.2,122.3,122.2,47.1,39.5,20.9 . \mathrm{IR}\left(\mathrm{KBr}, \mathrm{cm}^{-1}\right) v$ 3018, 2926, 2848, 1603, 1481, 743. HRMS (ESI): m/z $\left(\mathrm{M}^{+}\right)$calcd for $\mathrm{C}_{15} \mathrm{H}_{15} \mathrm{~N}: 209.1204$. Found 209.1206.

2-(2,3-dihydro-1H-inden-2-yl)-5-methylpyridine (Table 2, entry 10), a yellow oil. ${ }^{1} \mathrm{H}$ NMR $\left(500 \mathrm{MHz}, \mathrm{CDCl}_{3}\right): \delta 8.39(\mathrm{~d}, J=2.0 \mathrm{~Hz}, 1 \mathrm{H}), 7.42(\mathrm{dd}$, $J=8.0 \mathrm{~Hz}, 2.0 \mathrm{~Hz}, 1 \mathrm{H}), 7.28-7.21(\mathrm{~m}, 2 \mathrm{H}), 7.20-7.15(\mathrm{~m}, 2 \mathrm{H}), 7.13(\mathrm{~d}, J=$ $8.0 \mathrm{~Hz}, 1 \mathrm{H}), 3.92-3.78(\mathrm{~m}, 1 \mathrm{H}), 3.37(\mathrm{dd}, J=15.4 \mathrm{~Hz}, 8.4 \mathrm{~Hz}, 2 \mathrm{H}), 3.24$ (dd, $J=15.5 \mathrm{~Hz}, 8.8 \mathrm{~Hz}, 2 \mathrm{H}), 2.31(\mathrm{~s}, 3 \mathrm{H}) \cdot{ }^{13} \mathrm{C} \mathrm{NMR}\left(125 \mathrm{MHz}, \mathrm{CDCl}_{3}\right): \delta$ 161.5, 149.6, 142.9, 137.1, 130.6, 126.5, 124.4, 121.0, 47.0, 39.8, 18.1. IR $\left(\mathrm{KBr}, \mathrm{cm}^{-1}\right) \vee 3020,2934,2845,1600,1483,1459,743 . \mathrm{HRMS}(\mathrm{ESI}): \mathrm{m} / \mathrm{z}$ $\left(\mathrm{M}^{+}\right)$calcd for $\mathrm{C}_{15} \mathrm{H}_{15} \mathrm{~N}: 209.1204$. Found 209.1206.

2-(2,3-dihydro-1H-inden-2-yl)-5-methoxypyridine (Table 2, entry 11), a white solid. mp 65-67 ${ }^{\circ} \mathrm{C} .{ }^{1} \mathrm{H}$ NMR $\left(500 \mathrm{MHz}, \mathrm{CDCl}_{3}\right): \delta 8.27(\mathrm{dd}, J=2.5$ $\mathrm{Hz}, 1.0 \mathrm{~Hz}, 1 \mathrm{H}), 7.25-7.22(\mathrm{~m}, 2 \mathrm{H}), 7.20-7.12(\mathrm{~m}, 4 \mathrm{H}), 3.85(\mathrm{~s}, 3 \mathrm{H})$, 3.88-3.80 (m, 1H), $3.35(\mathrm{dd}, J=15.3 \mathrm{~Hz}, 8.3 \mathrm{~Hz}, 2 \mathrm{H}), 3.22(\mathrm{dd}, J=15.5 \mathrm{~Hz}$, $8.8 \mathrm{~Hz}, 2 \mathrm{H}) .{ }^{13} \mathrm{C} \mathrm{NMR}\left(125 \mathrm{MHz}, \mathrm{CDCl}_{3}\right): \delta$ 156.4, 154.0, 142.8, 136.4, 126.4, 124.3, 121.5, 121.3, 55.6, 46.5, 39.8. IR $\left(\mathrm{KBr}, \mathrm{cm}^{-1}\right) \vee 3011,2926$, 2849, 1492, 1456,1265, 1030, 744. HRMS (ESI): $\mathrm{m} / \mathrm{z}\left(\mathrm{M}^{+}\right)$calcd for $\mathrm{C}_{15} \mathrm{H}_{15} \mathrm{NO}: 225.1154$. Found 225.1155.

2-(2,3-dihydro-1H-inden-2-yl)-6-methylpyridine (Table 2 , entry 12), a yellow oil. ${ }^{1} \mathrm{H}$ NMR $\left(500 \mathrm{MHz}, \mathrm{CDCl}_{3}\right): \delta 7.48(\mathrm{t}, J=7.7 \mathrm{~Hz}, 1 \mathrm{H})$, 
7.26-7.22 (m, 2H), 7.20-7.15 (m, 2H), $7.00(\mathrm{dd}, J=12.6 \mathrm{~Hz}, 7.8 \mathrm{~Hz}, 2 \mathrm{H})$,

3.92-3.79 (m, 1H), $3.38(\mathrm{dd}, J=15.5 \mathrm{~Hz}, 8.4 \mathrm{~Hz}, 2 \mathrm{H}), 3.24(\mathrm{dd}, J=15.6 \mathrm{~Hz}$,

$8.5 \mathrm{~Hz}, 2 \mathrm{H}), 2.55(\mathrm{~s}, 3 \mathrm{H}) .{ }^{13} \mathrm{C} \mathrm{NMR}\left(125 \mathrm{MHz}, \mathrm{CDCl}_{3}\right): \delta$ 164.0, 157.7, $142.9,136.7,126.4,124.4,120.8,118.0,47.5,39.9,24.6$.

2-(2,3-dihydro-1H-inden-2-yl)-5-(trifluoromethyl)pyridine (Table 2, entry 13), a yellow solid. mp 74-76 ${ }^{\circ} \mathrm{C} .{ }^{1} \mathrm{H}$ NMR (500 $\mathrm{MHz}, \mathrm{CDCl}_{3}$ ): $\delta$ 8.87-8.81 (m, 1H), $7.84(\mathrm{dd}, J=8.2 \mathrm{~Hz}, 2.1 \mathrm{~Hz}, 1 \mathrm{H}), 7.35(\mathrm{~d}, J=8.2 \mathrm{~Hz}$, $1 \mathrm{H}), 7.30-7.23(\mathrm{~m}, 2 \mathrm{H}), 7.22-7.17(\mathrm{~m}, 2 \mathrm{H}), 3.99-3.91(\mathrm{~m}, 1 \mathrm{H}), 3.41(\mathrm{dd}, J=$ $15.7 \mathrm{~Hz}, 8.4 \mathrm{~Hz}, 2 \mathrm{H}), 3.29(\mathrm{dd}, J=15.6 \mathrm{~Hz}, 8.5 \mathrm{~Hz}, 2 \mathrm{H}) .{ }^{13} \mathrm{C}$ NMR (125 $\left.\mathrm{MHz}, \mathrm{CDCl}_{3}\right): \delta 168.5,146.2(\mathrm{q}, J=3.8 \mathrm{~Hz}), 142.2,133.5(\mathrm{q}, J=3.4 \mathrm{~Hz})$, 126.7, 124.4, $124.4(\mathrm{q}, J=32.5 \mathrm{~Hz}), 123.8\left(\mathrm{q}, J=270.1 \mathrm{~Hz}, \mathrm{CF}_{3}\right), 121.3$, 47.3, 39.6. IR $\left(\mathrm{KBr}, \mathrm{cm}^{-1}\right) \vee$ 3024, 2922, 2847, 1605, 1484, 1458, 1329, 747. HRMS (ESI): $\mathrm{m} / \mathrm{z}\left(\mathrm{M}^{+}\right)$calcd for $\mathrm{C}_{15} \mathrm{H}_{12} \mathrm{~F}_{3} \mathrm{~N}: 263.0922$. Found 263.0924 .

2-(2-((tert-butyldimethylsilyl)oxy)cyclopentyl)pyridine(Table 2, entry 14), a yellow oil. ${ }^{1} \mathrm{H}$ NMR (500 MHz, $\left.\mathrm{CDCl}_{3}\right): \delta 8.56(\mathrm{dd}, J=4.8 \mathrm{~Hz}, 0.8$ $\mathrm{Hz}, 1 \mathrm{H}), 7.55(\mathrm{td}, J=7.7 \mathrm{~Hz}, 1.9 \mathrm{~Hz}, 1 \mathrm{H}), 7.14(\mathrm{~d}, J=7.8 \mathrm{~Hz}, 1 \mathrm{H})$, 7.11-7.06 (m, 1H), $4.31(\mathrm{q}, J=7.4 \mathrm{~Hz}, 1 \mathrm{H}), 3.06-2.95(\mathrm{~m}, 3 \mathrm{H}), 2.12-1.93$ (m, 2H), 1.89-1.75 (m, 1H), 1.71-1.60 (m, 1H), $0.75(\mathrm{~s}, 9 \mathrm{H}),-0.20(\mathrm{~s}, 3 \mathrm{H})$, $-0.29(\mathrm{~s}, 3 \mathrm{H}) .{ }^{13} \mathrm{C} \mathrm{NMR}\left(125 \mathrm{MHz}, \mathrm{CDCl}_{3}\right): \delta 163.2,149.4,135.8,123.9$, $121.2,80.6,56.3,35.1,30.2,25.7,21.9,18.0,-5.10,-5.20 . \operatorname{IR}\left(\mathrm{KBr}, \mathrm{cm}^{-1}\right) v$ 3006, 2928, 2856, 1590, 1472, 1113, 836, 775. GC-MS (EI): m/z $\left(\mathrm{M}^{+}\right)$ 
calcd for $\mathrm{C}_{16} \mathrm{H}_{27} \mathrm{NOSi}$ : 277.2. Found 277.2.

3-(pyridin-2-yl)butyl benzoate (Table 2, entry 15), a white solid. mp 120-122 ${ }^{\circ} \mathrm{C} .{ }^{1} \mathrm{H}$ NMR $\left(500 \mathrm{MHz}, \mathrm{CDCl}_{3}\right): \delta 8.58-8.54(\mathrm{~m}, 1 \mathrm{H}), 8.01-7.95(\mathrm{~m}$, 2H), $7.59(\mathrm{td}, J=7.7 \mathrm{~Hz}, 1.8 \mathrm{~Hz}, 1 \mathrm{H}), 7.57-7.52(\mathrm{~m}, 1 \mathrm{H}), 7.46-7.39(\mathrm{~m}, 2 \mathrm{H})$, $7.16(\mathrm{~d}, J=7.8 \mathrm{~Hz}, 1 \mathrm{H}), 7.13-7.08(\mathrm{~m}, 1 \mathrm{H}), 4.35-4.27(\mathrm{~m}, 1 \mathrm{H}), 4.26-4.20$ (m, 1H), 3.18-3.07 (m, 1H), 2.35-2.25 (m, 1H), 2.14-2.09 (m, 1H), $1.36(\mathrm{~d}, J$ $=7.0 \mathrm{~Hz}, 3 \mathrm{H}) .{ }^{13} \mathrm{C} \mathrm{NMR}\left(125 \mathrm{MHz}, \mathrm{CDCl}_{3}\right): \delta 166.5,165.1,149.4,136.5$, $132.8,130.3,129.5,128,3121.9,121.4,63.4,38.9,35.4,21.1 . \mathrm{IR}(\mathrm{KBr}$, $\left.\mathrm{cm}^{-1}\right) \vee 3006,2963,2925,1717,1590,1474,1276,1114,712$. HRMS (ESI): $\mathrm{m} / \mathrm{z}\left(\mathrm{M}^{+}\right)$calcd for $\mathrm{C}_{16} \mathrm{H}_{17} \mathrm{NO}_{2}: 255.1259$. Found 225.1261.

3-(pyridin-2-yl)butyl 4-methoxybenzoate (Table 2, entry 16), a white solid. mp 156-158 ${ }^{\circ} \mathrm{C} .{ }^{1} \mathrm{H}$ NMR (500 MHz, $\left.\mathrm{CDCl}_{3}\right): \delta 8.58-8.51(\mathrm{~m}, 1 \mathrm{H})$, 7.98-7.88 (m, 2H), $7.58(\mathrm{td}, J=7.7 \mathrm{~Hz}, 1.9 \mathrm{~Hz}, 1 \mathrm{H}), 7.15(\mathrm{~d}, J=7.8 \mathrm{~Hz}$, 1H), 7.13-7.07 (m, 1H), 6.93-6.86 (m, 2H), 4.30-4.23 (m, 1H), 4.22-4.16 (m, 1H), $3.85(\mathrm{~s}, 3 \mathrm{H}), 3.19-3.03(\mathrm{~m}, 1 \mathrm{H}), 2.33-2.20(\mathrm{~m}, 1 \mathrm{H}), 2.13-2.01(\mathrm{~m}, 1 \mathrm{H})$, $1.36(\mathrm{~d}, J=7.0 \mathrm{~Hz}, 3 \mathrm{H}) .{ }^{13} \mathrm{C} \mathrm{NMR}\left(125 \mathrm{MHz}, \mathrm{CDCl}_{3}\right): \delta 166.3,165.2$, $163.2,149.4,136.4,131.5,122.8,121.9,121.3,113.5,63.1,55.4,38.9,35.5$, 21.1. IR $\left(\mathrm{KBr}, \mathrm{cm}^{-1}\right) \vee 3006,2962,2928,2843,1710,1606,1511,1277$, 1257, 1168, 771. HRMS (ESI): m/z $\left(\mathrm{M}^{+}\right)$calcd for $\mathrm{C}_{16} \mathrm{H}_{17} \mathrm{NO}_{2}: 285.1365$. Found 285.1368. 


\section{III. ${ }^{1} \mathrm{H}$ NMR and ${ }^{13} \mathrm{C}$ NMR spetra}<smiles>[As]N1CCC(C2=NCCN2)CC1</smiles>

\section{(Table 2, entry 3)}
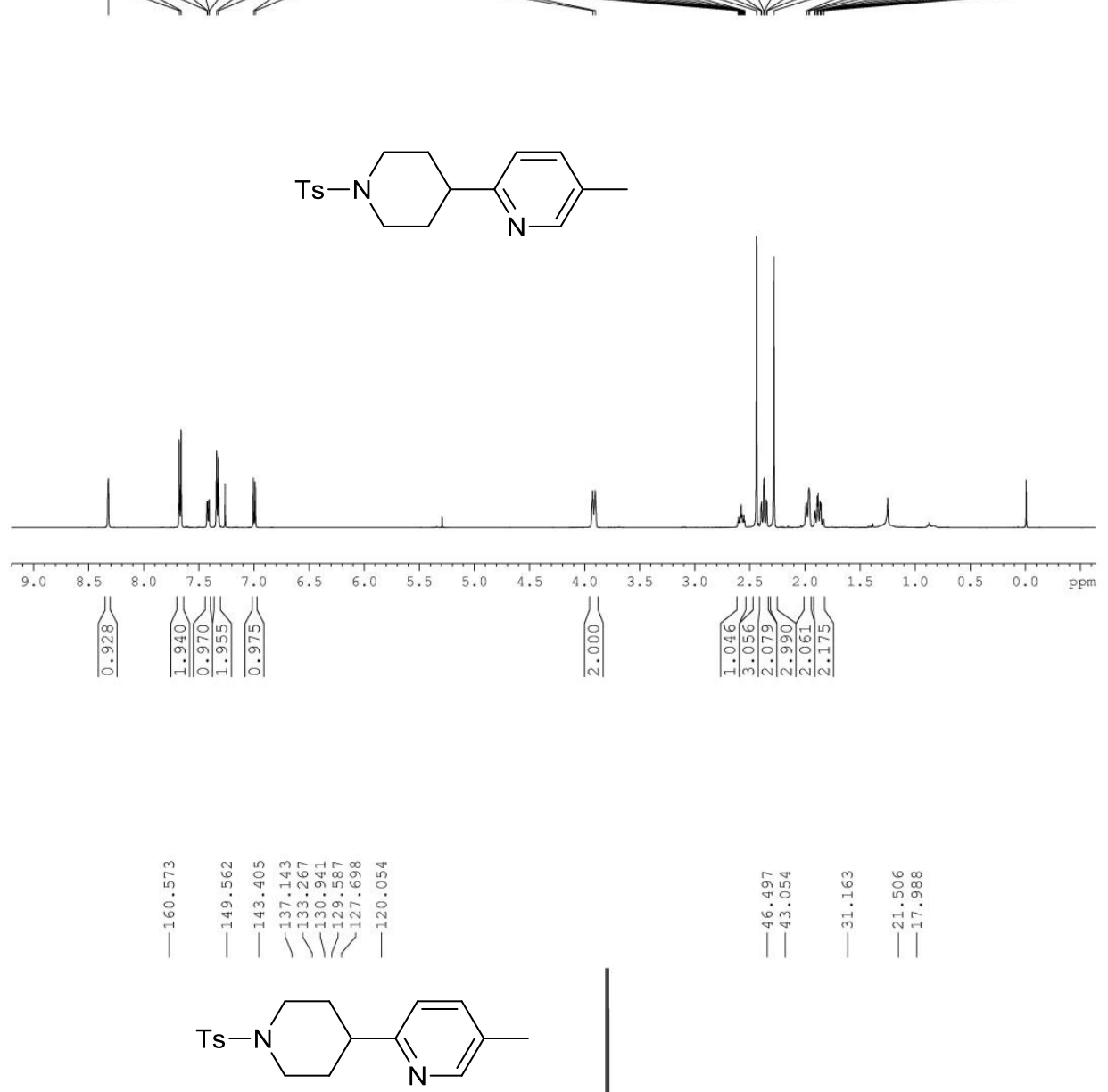

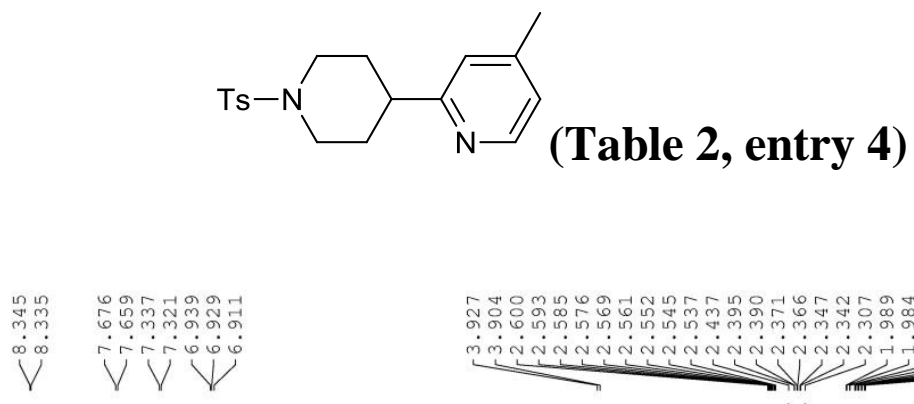

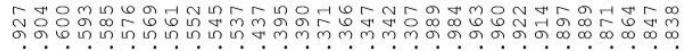

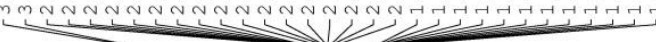
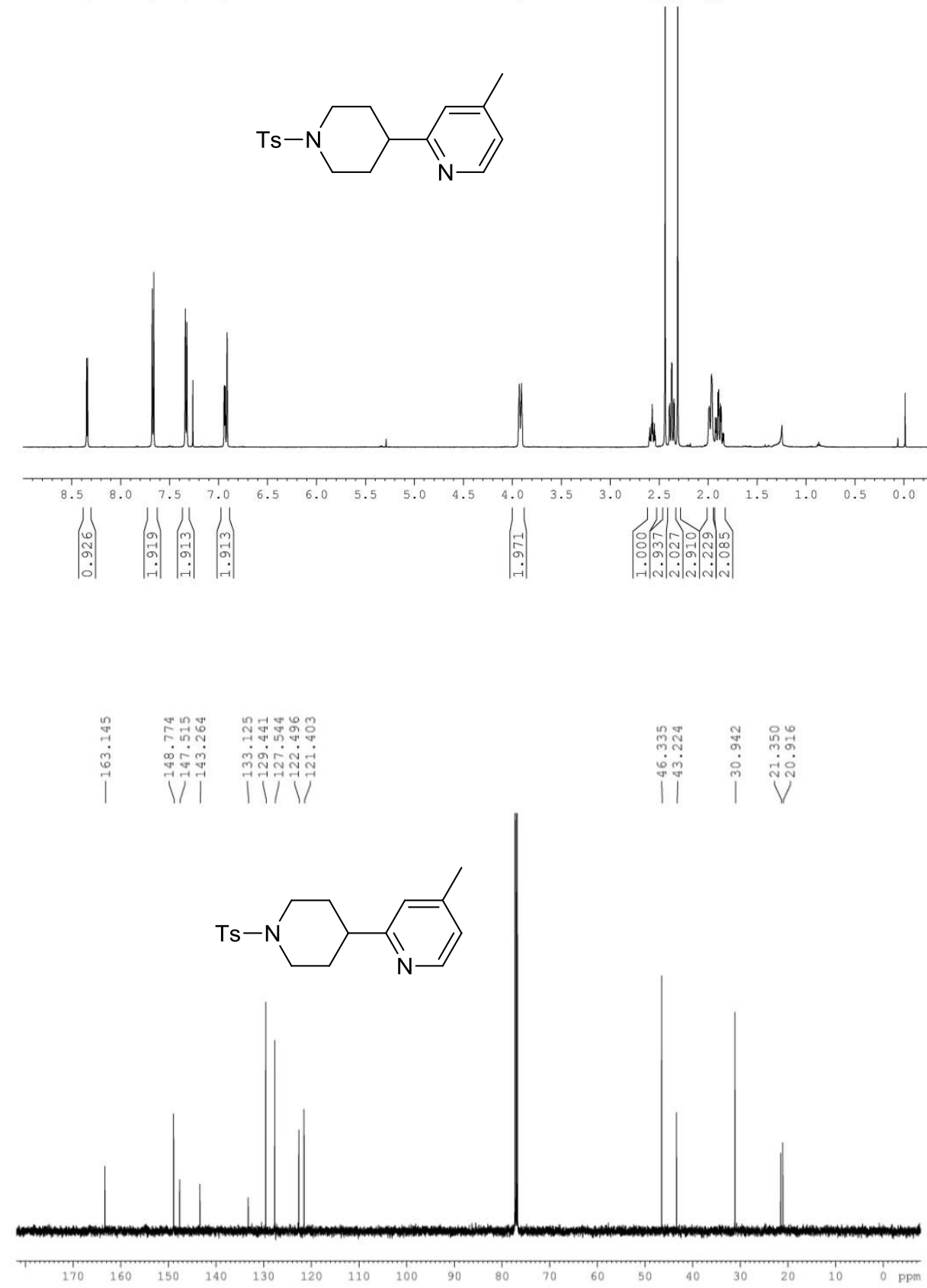


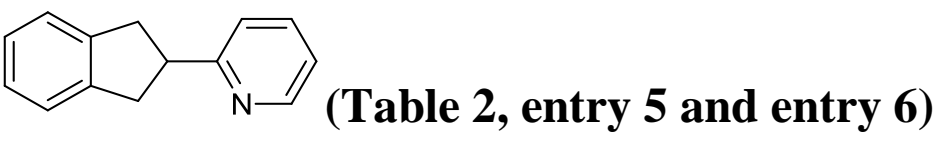

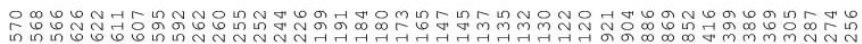

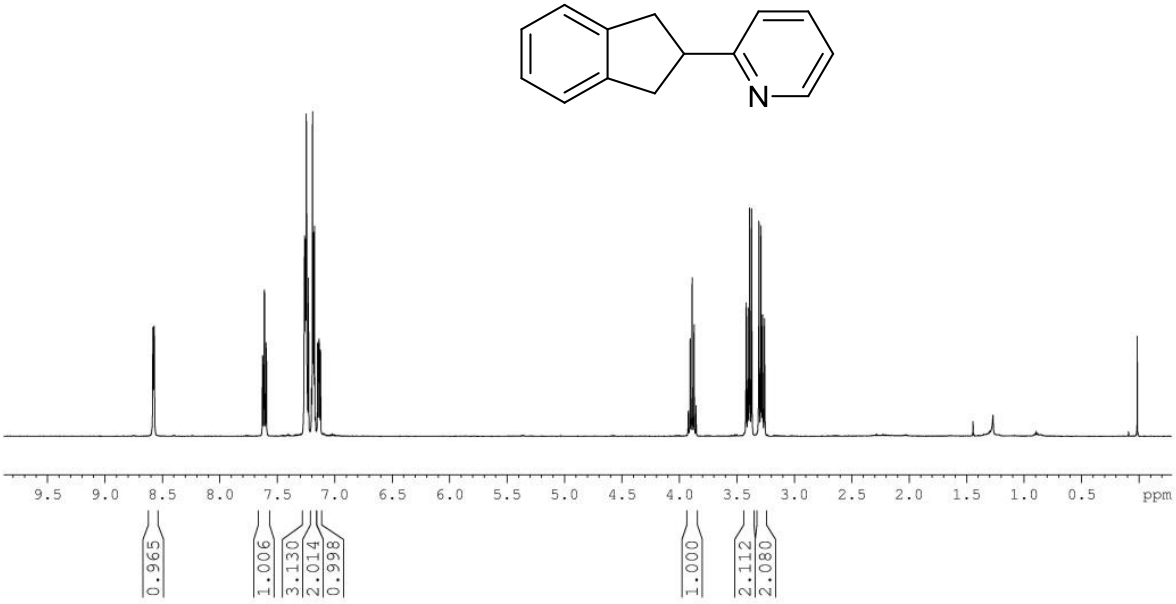

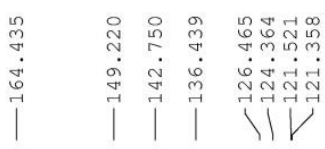<smiles>c1ccc(C2Cc3ccccc3C2)nc1</smiles>

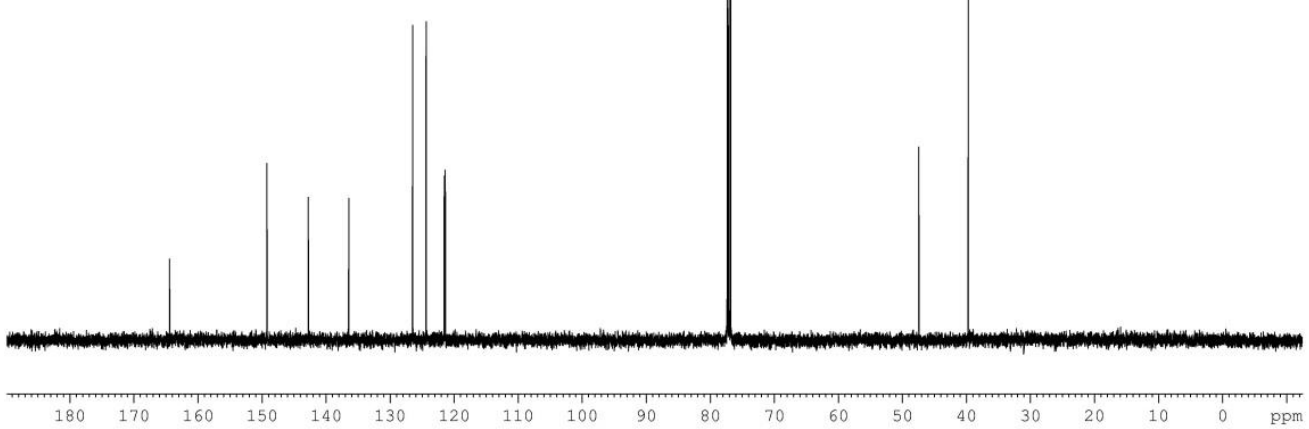


<smiles>c1cncc(C2Cc3ccccc3C2)c1</smiles>

(Table 2, entry 7)

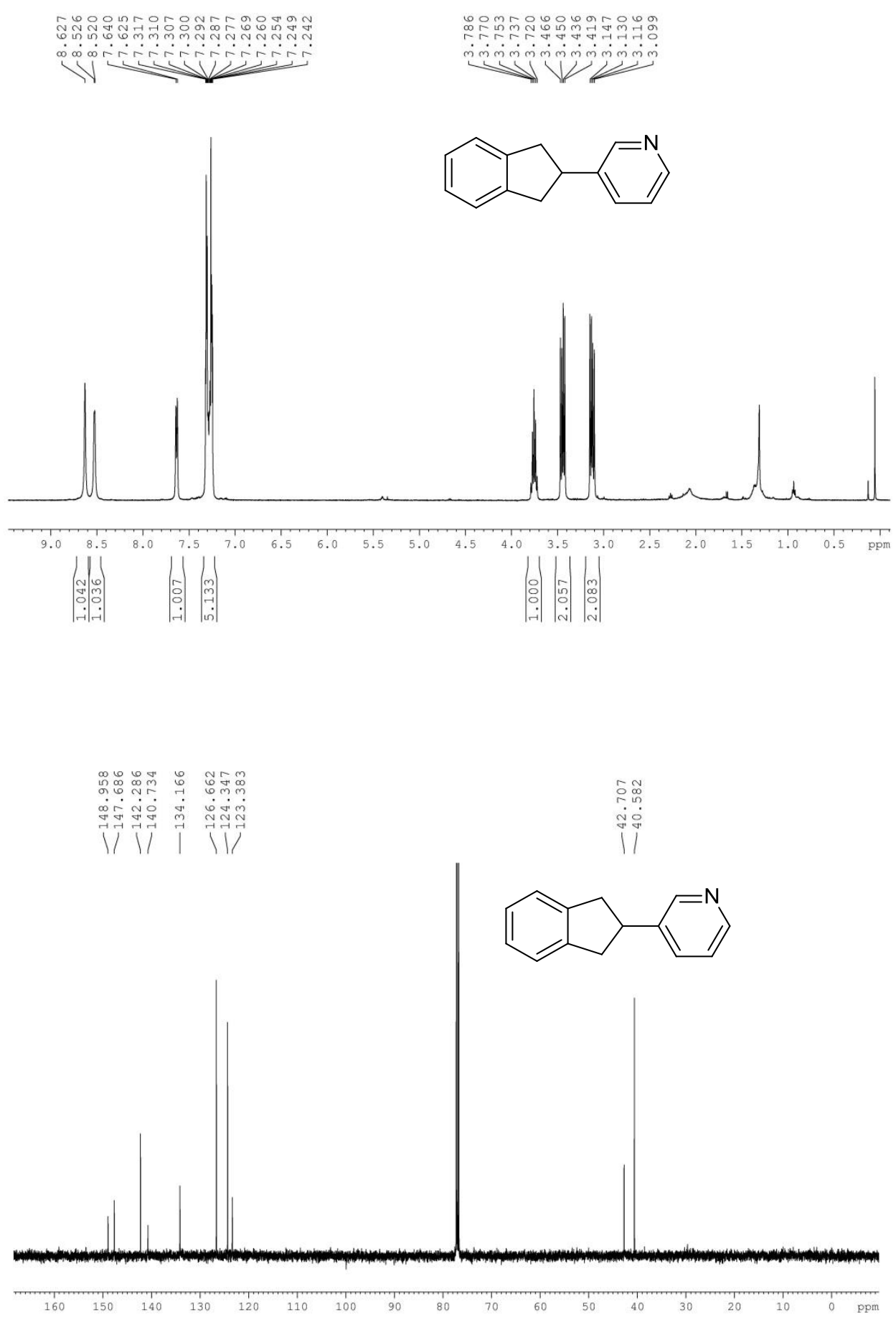



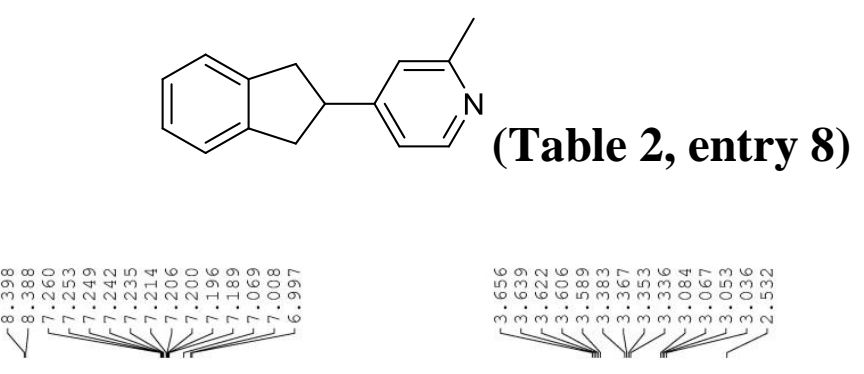

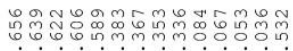

nंलेंm.

证
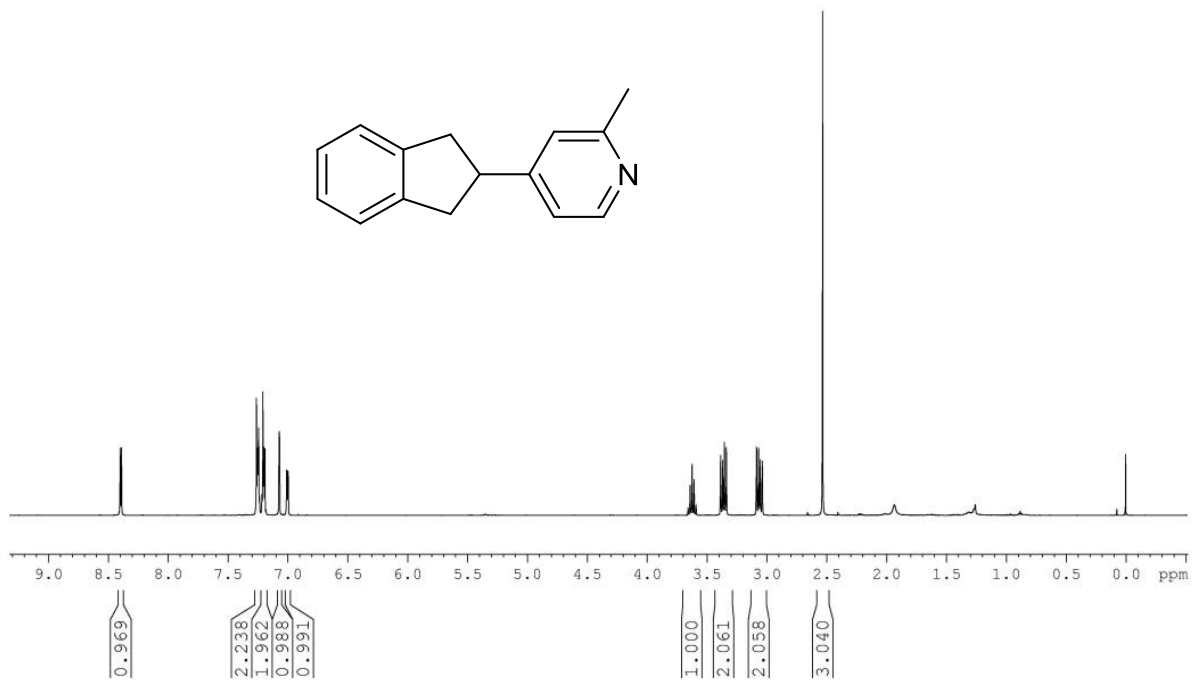

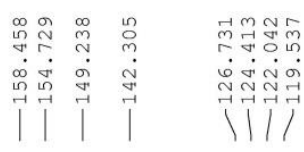

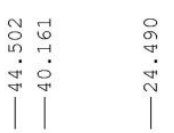

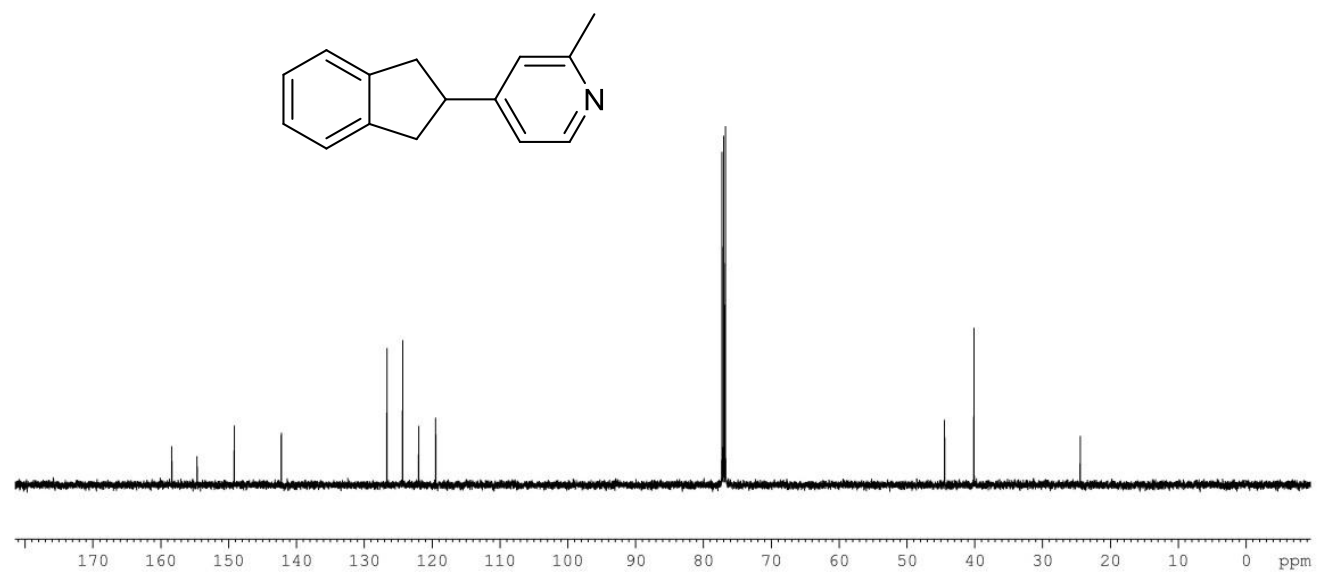




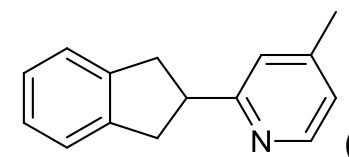

\section{(Table 2, entry 9)}
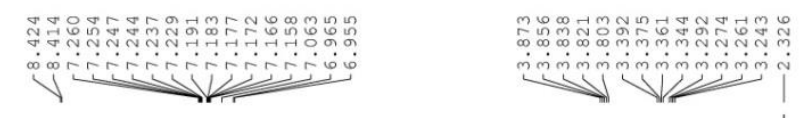<smiles>Cc1ccnc(C2Cc3ccccc3C2)c1</smiles>
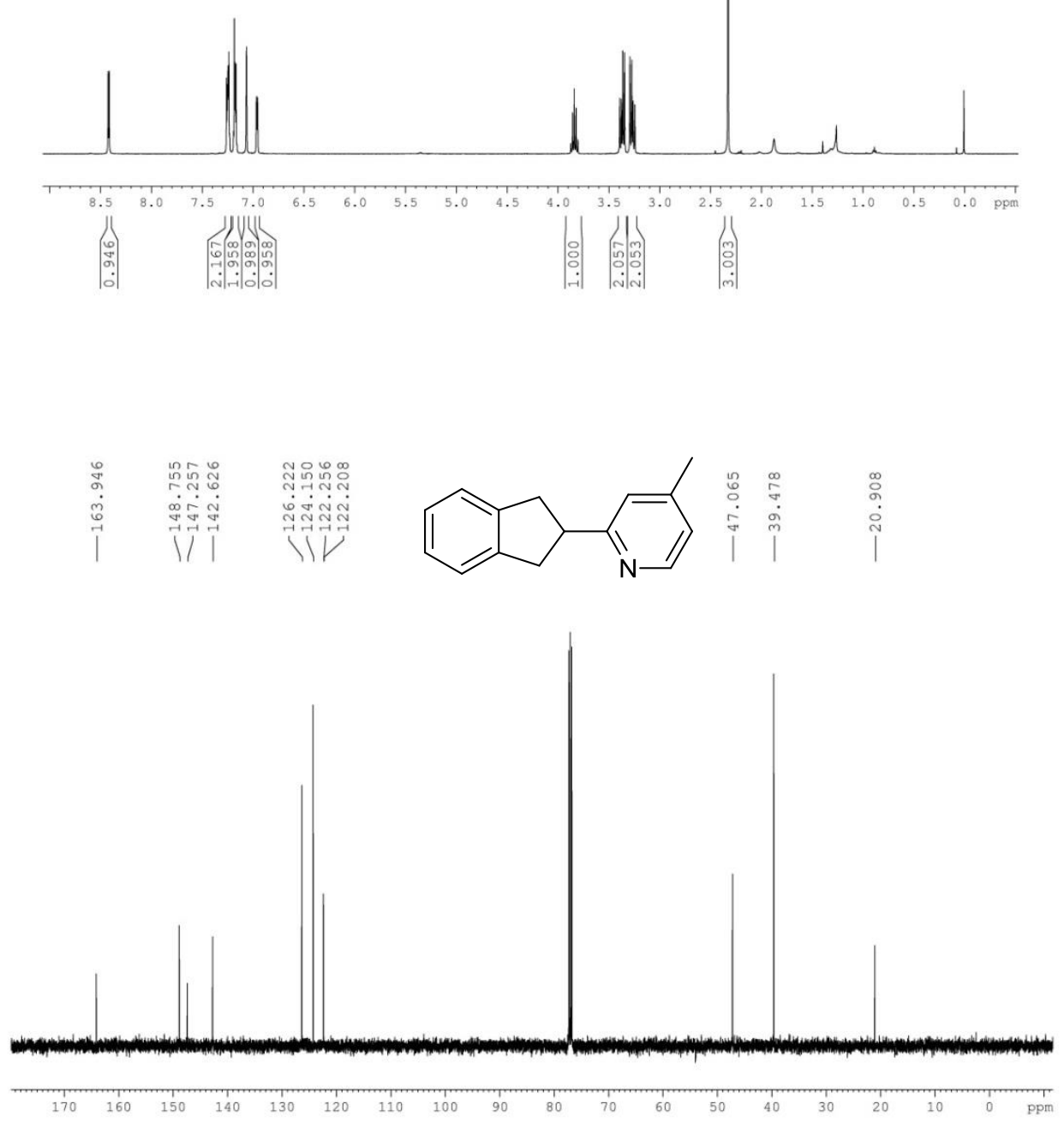


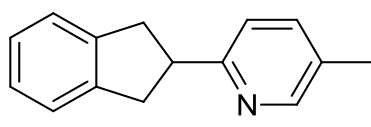

\section{(Table 2, entry 10)}
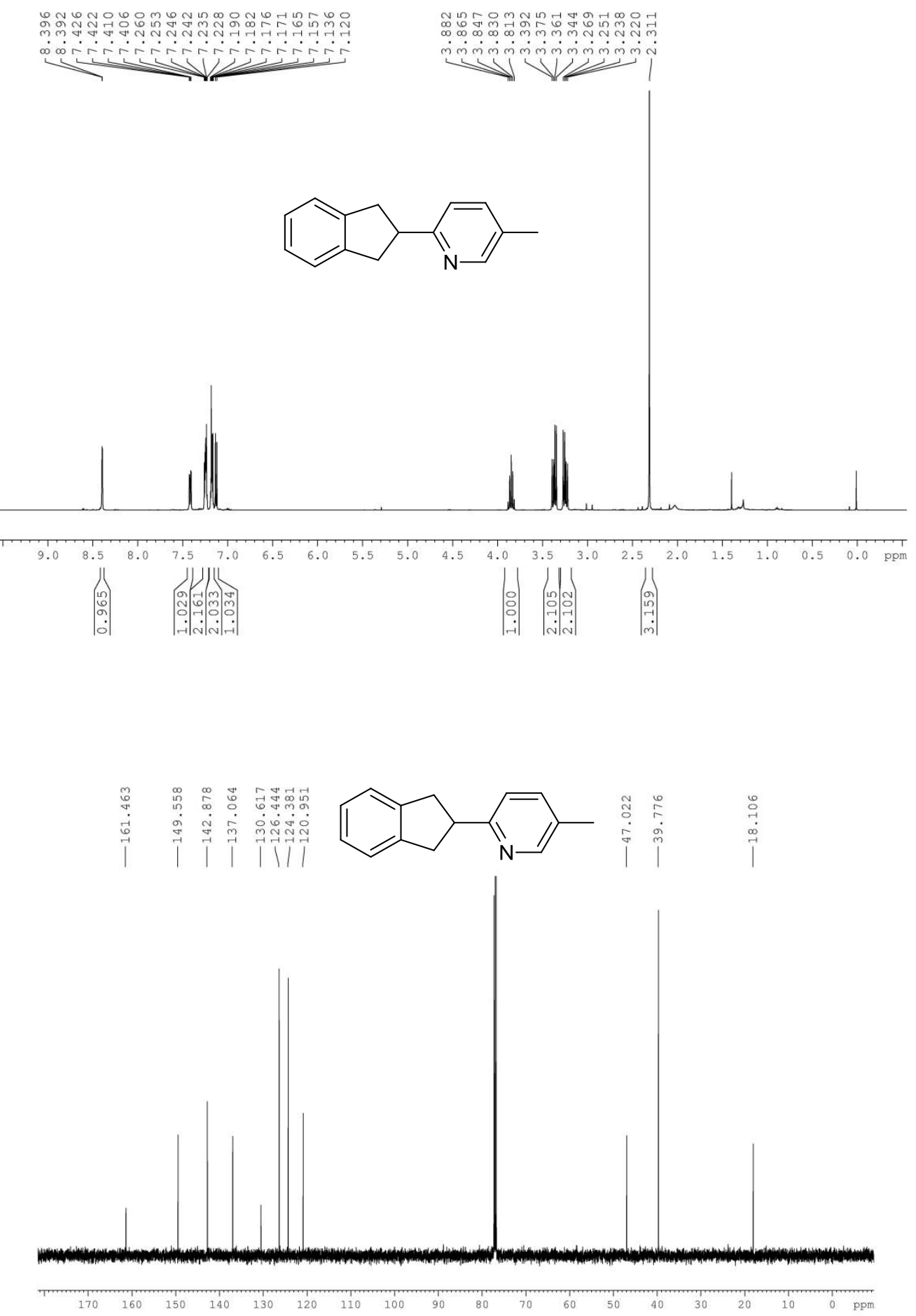
<smiles>COc1ccc(C2Cc3ccccc3C2)nc1</smiles>

(Table 2, entry 11)
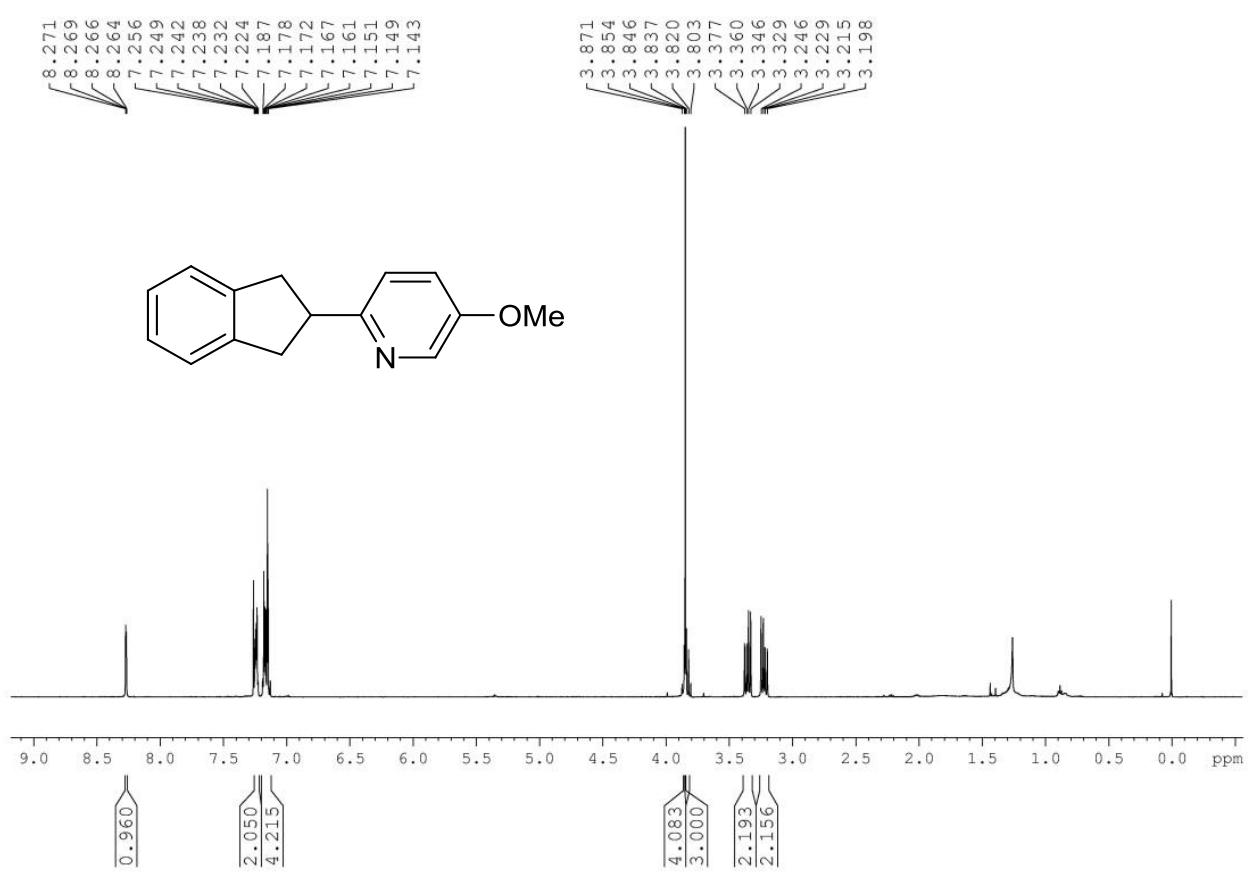

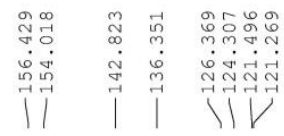<smiles>COc1ccc(C2Cc3ccccc3C2)nc1</smiles>

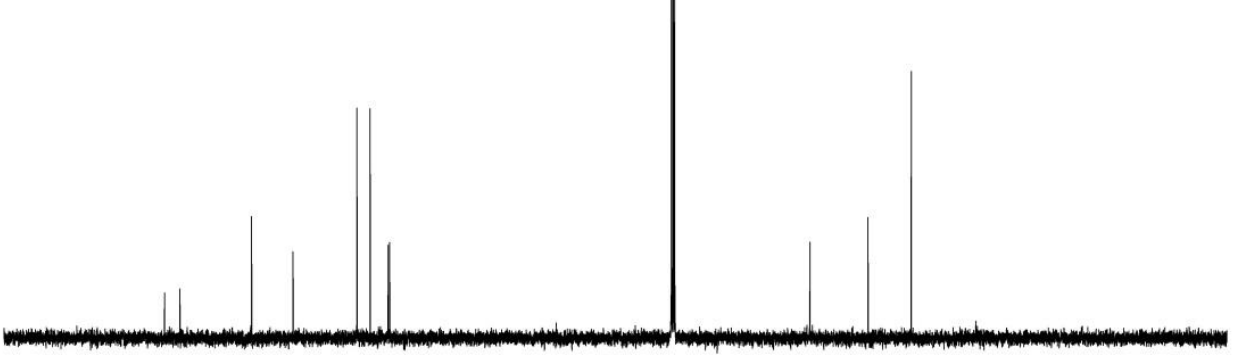



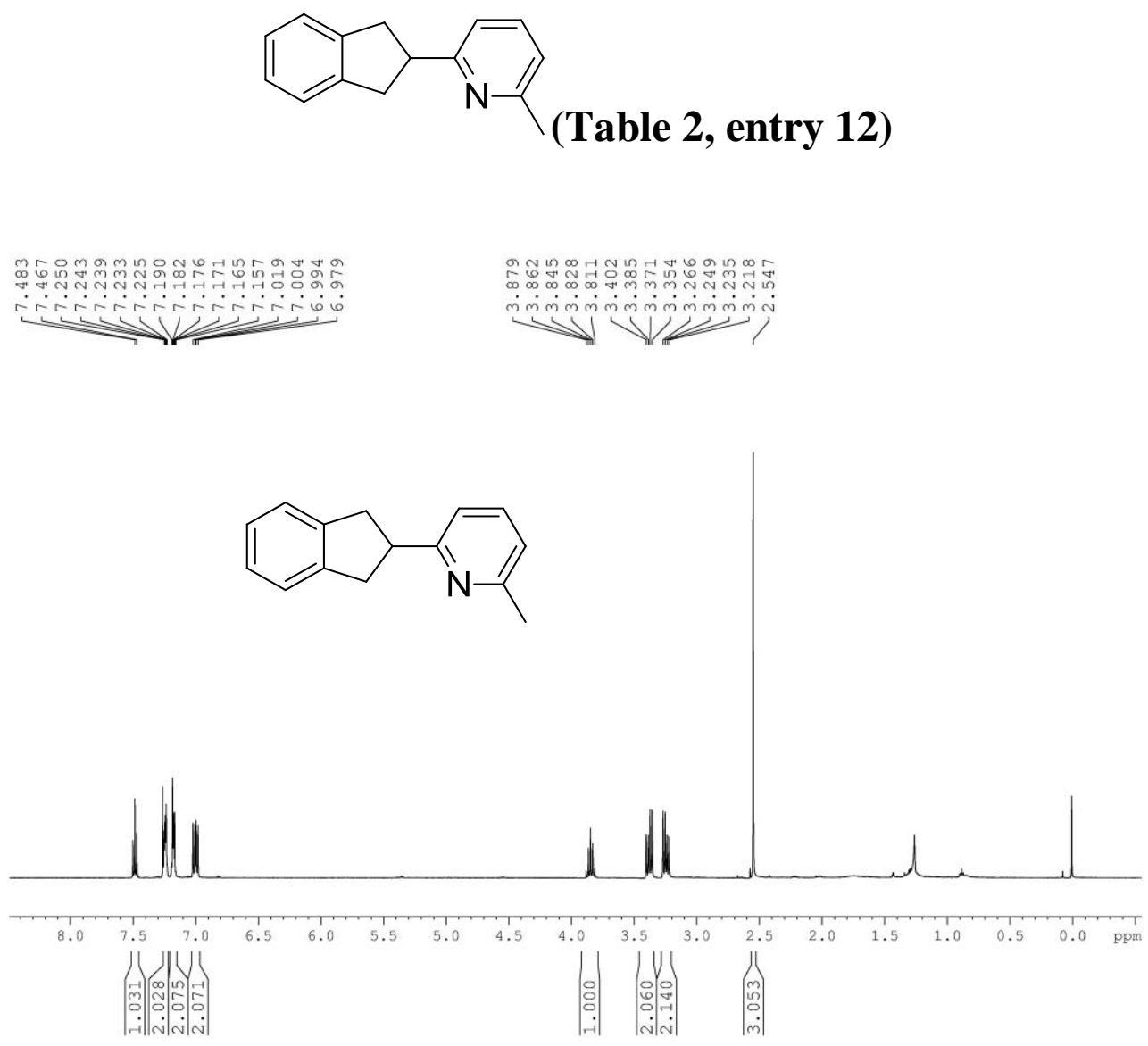

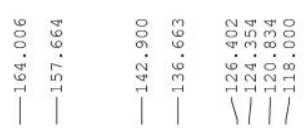
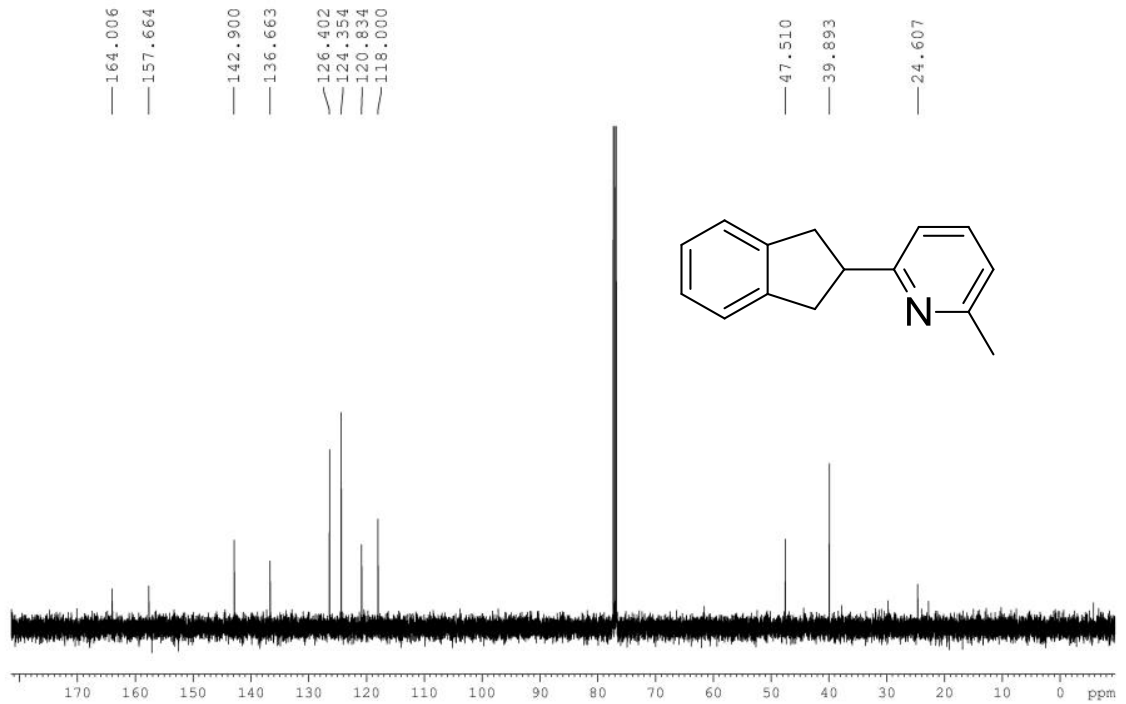
<smiles>FC(F)(F)c1ccc(C2Cc3ccccc3C2)nc1</smiles>

(Table 2, entry 13)

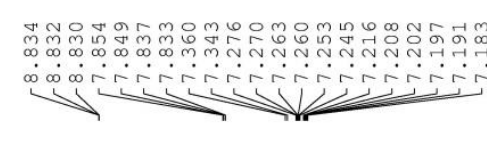

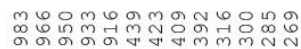

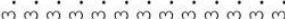

$\underbrace{m i n} \underbrace{m}$
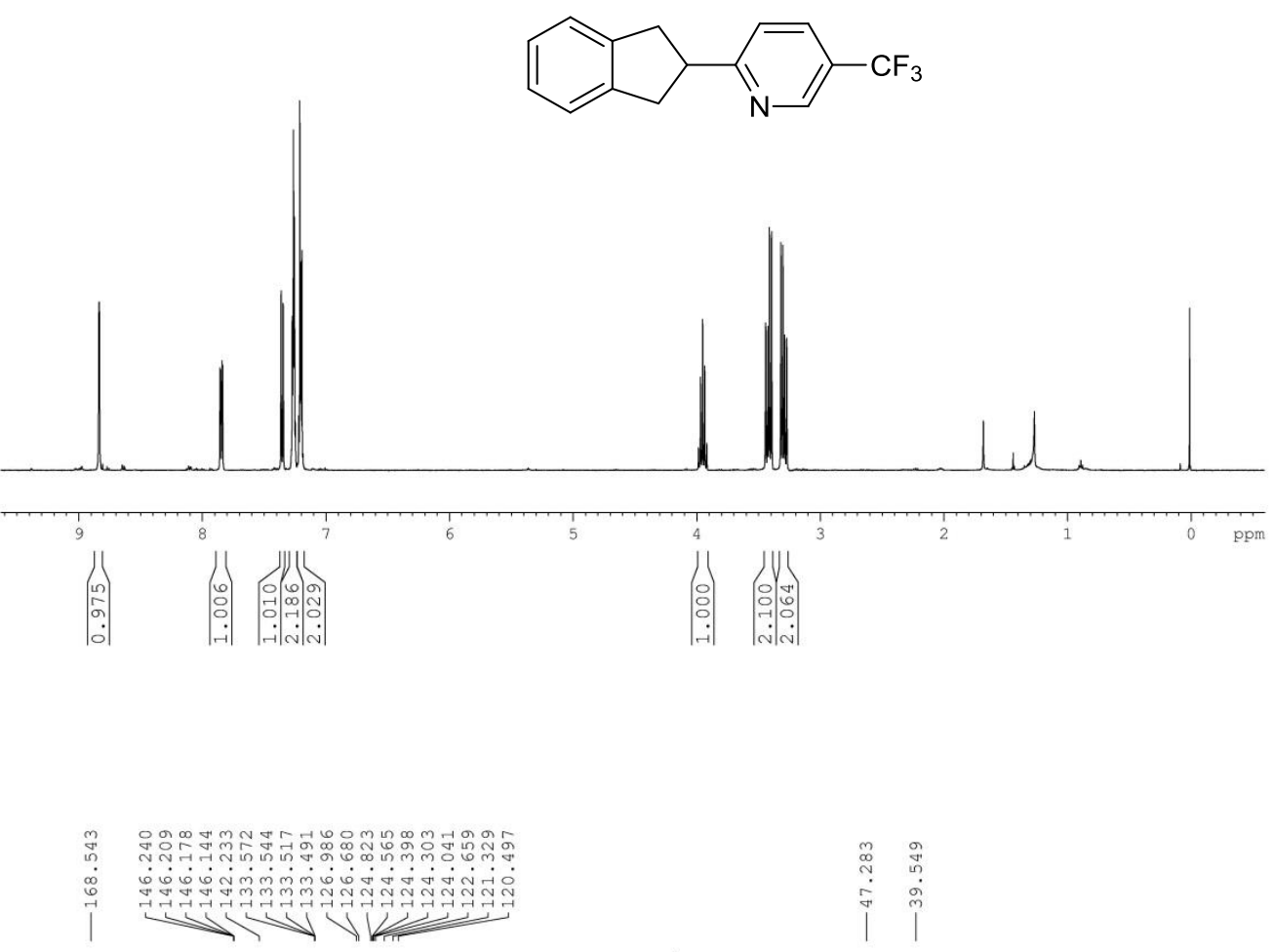

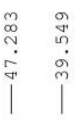

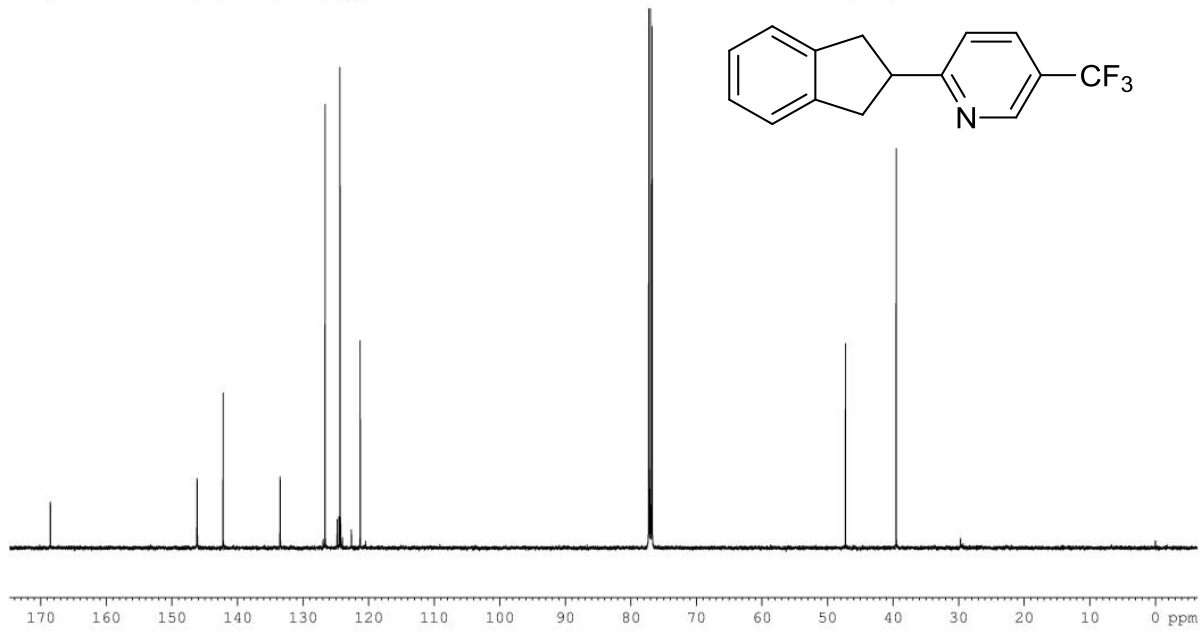



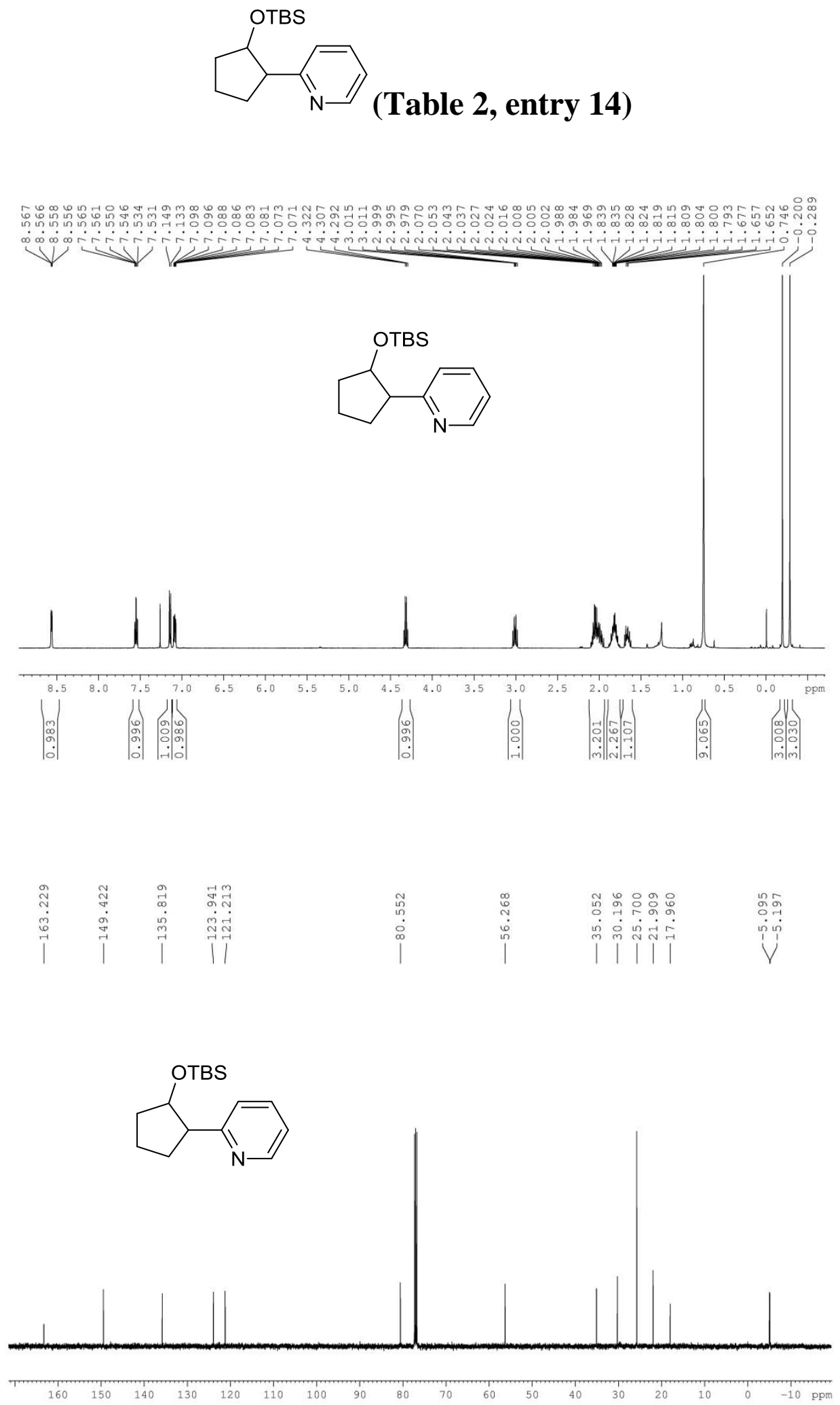

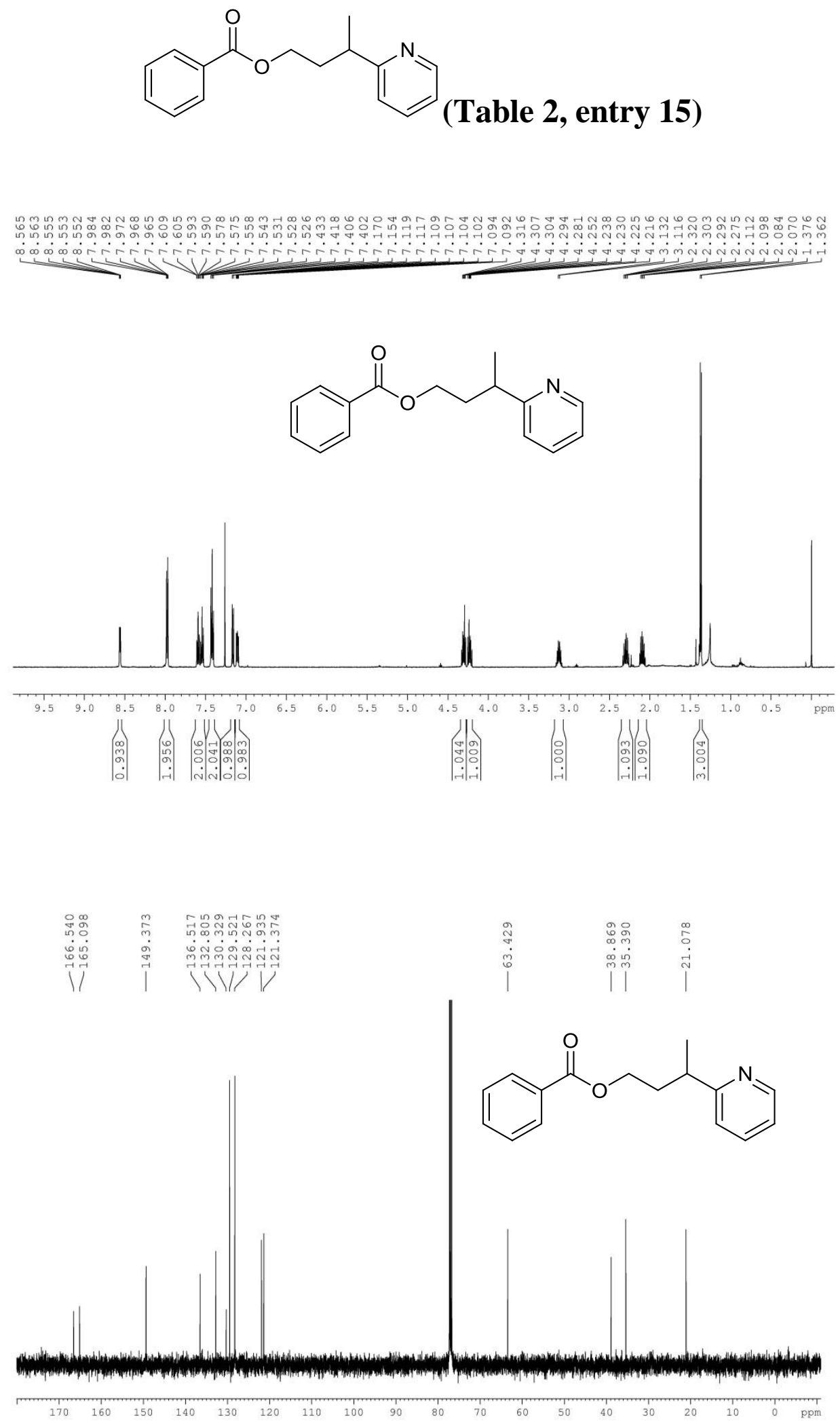


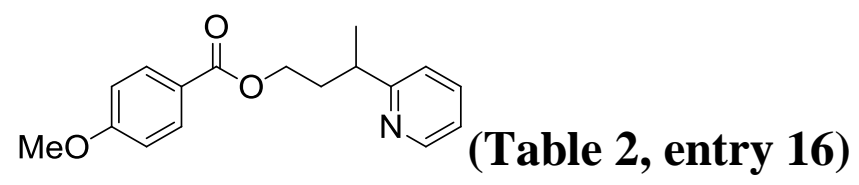

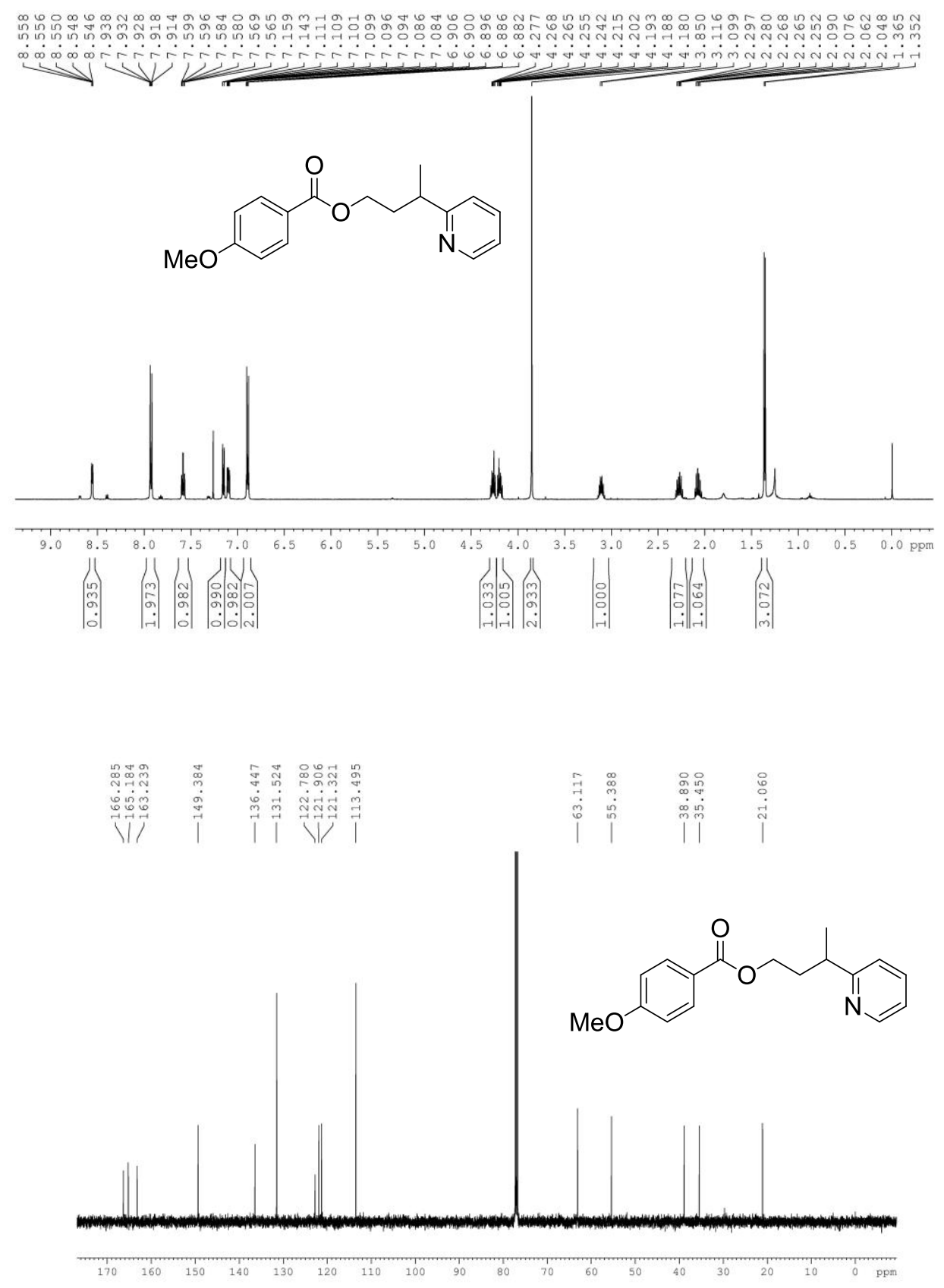

